\title{
Article \\ A Nanoplasmonic-Based Biosensing Approach for Wide-Range and Highly Sensitive Detection of Chemicals
}

\author{
Francesco Arcadio ${ }^{1}{ }^{\oplus}$, Luigi Zeni ${ }^{1} \oplus$, Aldo Minardo ${ }^{1}{ }^{\circledR}$, Caterina Eramo $^{1}$, Stefania Di Ronza ${ }^{1}$, Chiara Perri $^{1}$, \\ Girolamo D'Agostino ${ }^{2}$, Guido Chiaretti ${ }^{2}$, Giovanni Porto ${ }^{2}$ and Nunzio Cennamo ${ }^{1, *(\mathbb{D}}$ \\ 1 Department of Engineering, University of Campania Luigi Vanvitelli, Via Roma, 29, 81031 Aversa, Italy; \\ francesco.arcadio@unicampania.it (F.A.); luigi.zeni@unicampania.it (L.Z.); \\ aldo.minardo@unicampania.it (A.M.); caterina.eramo@unicampania.it (C.E.); \\ stefania.dironza@unicampania.it (S.D.R.); chiara.perri@unicampania.it (C.P.) \\ 2 Moresense Srl., Filarete Foundation, Viale Ortles 22/4, 20139 Milan, Italy; g.dagostino@moresense.tech (G.D.); \\ g.chiaretti@moresense.tech (G.C.); g.porto@moresense.tech (G.P.) \\ * Correspondence: nunzio.cennamo@unicampania.it; Tel.: +39-081-5010-379
}

\section{check for} updates

Citation: Arcadio, F.; Zeni, L.; Minardo, A.; Eramo, C.; Di Ronza, S.; Perri, C.; D'Agostino, G.; Chiaretti, G.; Porto, G.; Cennamo, N. A

Nanoplasmonic-Based Biosensing Approach for Wide-Range and Highly Sensitive Detection of Chemicals. Nanomaterials 2021, 11, 1961. https:// doi.org/10.3390/nano11081961

Academic Editors: Marcin Runowski and Maria Losurdo

Received: 5 June 2021

Accepted: 26 July 2021

Published: 30 July 2021

Publisher's Note: MDPI stays neutral with regard to jurisdictional claims in published maps and institutional affiliations.

Copyright: (c) 2021 by the authors. Licensee MDPI, Basel, Switzerland. This article is an open access article distributed under the terms and conditions of the Creative Commons Attribution (CC BY) license (https:// creativecommons.org/licenses/by/ $4.0 /)$.

\begin{abstract}
In a specific biosensing application, a nanoplasmonic sensor chip has been tested by an experimental setup based on an aluminum holder and two plastic optical fibers used to illuminate and collect the transmitted light. The studied plasmonic probe is based on gold nanograting, realized on the top of a Poly (methyl methacrylate) (PMMA) chip. The PMMA substrate could be considered as a transparent substrate and, in such a way, it has been already used in previous work. Alternatively, here it is regarded as a slab waveguide. In particular, we have deposited upon the slab surface, covered with a nanograting, a synthetic receptor specific for bovine serum albumin (BSA), to test the proposed biosensing approach. Exploiting this different experimental configuration, we have determined how the orientation of the nanostripes forming the grating pattern, with respect to the direction of the input light (longitudinal or orthogonal), influences the biosensing performances. For example, the best limit of detection (LOD) in the BSA detection that has been obtained is equal to $23 \mathrm{pM}$. Specifically, the longitudinal configuration is characterized by two observable plasmonic phenomena, each sensitive to a different BSA concentration range, ranging from $\mathrm{pM}$ to $\mu \mathrm{M}$. This aspect plays a key role in several biochemical sensing applications, where a wide working range is required.
\end{abstract}

Keywords: nanoplasmonic sensors; slab waveguides; plastic optical fibers; biochemical sensors; optical sensors; e-beam lithography

\section{Introduction}

Planar waveguide-based sensors have shown great potential in several application fields and represent an active research area. The development of these devices has mainly been boosted by the increasing need for rapid and automated devices to operate in several areas [1-9]. In particular, these kinds of platforms can also be used to exploit Surface Plasmon Resonance (SPR) and Localized Surface Plasmon Resonance (LSPR) phenomena in order to achieve excellent performances in biosensing applications [10-17].

In the scientific literature, several configurations exist, based on planar slab waveguide integrated on silicon (Si)-based substrate used for multiple applications, like gas sensing [18-22], magnetic field sensing [23,24], biological and chemical species detection [2,6,25-27], and so on These platforms take advantage of the technological and fabrication processes of the microelectronic industry. However, the main disadvantages of this approach are related to the difficulties connected to those techniques in realizing thick layers, despite the several efficient solutions that have been proposed during the last years [28]. Moreover, when planar slab waveguides based on silicon are applied, the technology used to realize them could be very expensive, especially in prototype realization. More specifically, when considering plasmonic sensors, the 
number of modes (depending on the guiding layer thickness) propagating into the waveguide plays a fundamental role in the performance of the conceived sensor. In particular, in the last years, multimodal waveguides have been preferred because, even if they usually show a non-optimum signal to noise ratio (SNR), they present a better sensitivity than monomodal waveguides $[29,30]$. This aspect is crucial if related to biochemical sensing applications, where high sensitivity is required to detect as low a concentration as possible of the analyte of interest.

In any case, the trade-off between SNR and sensitivity has been largely investigated and it is one of the reasons why, for instance, large core diameter plastic optical fibers (POFs) are preferred instead of monomodal silica ones when realizing SPR sensors based on optical fibers [31-34]. So, multimode polymer slab waveguides have also drawn the scientific community's attention so far, showing excellent performances and results $[7,14,35,36]$.

We exploited a multimode slab waveguide based on a Poly(methyl methacrylate) substrate to realize a nanoplasmonic biosensor. This PMMA chip has already shown its capability to be used as a plasmonic sensor, as reported in [14]. In particular, the PMMA chip is able to ensure an appropriate number of angles that satisfy the SPR condition at the interface between the metallic layer and the dielectric medium [14]. For this reason, this SPR probe has also been successfully tested in chemical analysis, like in the furfural detection by a specific biomimetic receptor [37]. Despite this, the overall performances of the SPR sensor based on the PMMA slab waveguide are similar to an SPR D-shaped POF sensor [38]. Actually, the most valuable advantage in its usage is mainly related to the very simple fabrication process that includes only a $60 \mathrm{~nm}$ thick gold deposition step upon the PMMA substrate [14].

In order to exploit the maximum potential of this type of plastic slab waveguide chip and take full advantage of its dimension $(10 \mathrm{~mm} \times 10 \mathrm{~mm} \times 0.5 \mathrm{~mm})$, fully compliant to the modern holder of electron beam lithography (EBL) systems (e.g., Zeiss Supra v35-Raith Elphy Quantum system), we conceived and tested a plasmonic sensor based on a gold nanograting (GNG) fabricated on the top of this PMMA chip [39]. In particular, as proof of concept, we functionalized the nano groove surface with a synthetic receptor specific for the bovine serum albumin (BSA), and we tested its biosensing capabilities by using an experimental setup described in [39]. In particular, in [39] the PMMA substrate has been considered a transparent substrate instead of a waveguide. Moreover, a similar nanostructure has also demonstrated an excellent solution to realize Surface-enhanced Raman scattering (SERS) active substrates [40].

Interesting work has described the excellent progress in developing novel surface functionalization strategies and the formation of optically and mechanically stable LSPR sensors [41].

In this work, we used a different approach by considering the PMMA chip not like a transparent substrate but as a slab waveguide to excite the plasmonic phenomena. We functionalized the nanograting surface with a synthetic receptor specific for BSA to compare the sensor performances with other sensor configurations. In such a way, we first established how, in this sensor configuration, the nanostripes' orientation with respect to the direction of the input light beam (longitudinal or orthogonal) influences the biosensing capabilities. Moreover, we established that two distinct plasmonic phenomena can widen out the operating range relative to the BSA detection when considering the longitudinal configuration. The proposed experimental setup includes an aluminum holder that contains the PMMA-GNG chip and two POF patches used to connect the holder with a white light source and a spectrometer. Finally, we also carried out a comparative analysis in terms of limit of detection (LOD) with an SPR probe based on POF and functionalized with the same receptor. In this work, exploiting the proposed setup, we changed the angle of the incident light with respect to [39] in the plasmonic phenomena obtaining different performances. In particular, we used the best configuration obtained in [39] in terms of geometric parameters of the GNG. We do not present any parametric optimization of the device in numerical and experimental results, as reported in [39], even if the geometric parameter variation influences the performances. In fact, this work aims to compare the performances of the proposed setup with those proposed in [39]. 


\section{Materials and Methods}

\subsection{Plasmonic Sensor Fabrication}

To compare the results with those obtained exploiting a different sensor configuration [39], we used the same nanoplasmonic chip reported in [39].

In particular, each stripe presents a width equal to about $400 \mathrm{~nm}$, whereas the mutual spacing is equal to about $615 \mathrm{~nm}$, which means a grating period equal to about $1 \mu \mathrm{m}$.

The device fabrication steps are schematically recalled in Figure 1a. In summary, to realize the nanoplasmonic chip, the starting sample consists of a $10 \mathrm{~mm} \times 10 \mathrm{~mm} \times 0.5 \mathrm{~mm}$ PMMA slab waveguide (GoodFellow, Huntingdon, England) where, on its surface, a positive PMMA e-beam resist (AR-P 679.04, AllResist GmbH, Strausberg, Germany) is deposited through a spin coater running at $6000 \mathrm{rpm}$ for one minute (a film with a final thickness of about $220 \mathrm{~nm}$ ). The exposition process, carried out by using an electron beam lithography system (Zeiss Supra v35-Raith Elphy Quantum, Oberkochen, Germany), takes place in a total area of $1 \mathrm{~mm}^{2}(1 \mathrm{~mm} \times 1 \mathrm{~mm})$ at the center of the slab waveguide (see Figure 1b). The final steps consist of developing the exposed resist and depositing a $40 \mathrm{~nm}$ thick gold film through a sputtering machine (BalTec SCD 500, Schalksmühle, Germany). The cross section of the realized plasmonic GNG-based chip with the relative dimensions is shown in Figure 1c,d reports a Scanning Electron Microscope (SEM) image of the fabricated nanograting.

In this work, we did not carry out atomic force microscopy images and topographic profiles of the deposited gold film. So, even if the roughness of the gold film influences the sensitivity of the plasmonic sensor, we could not consider this aspect to modify the sensor performances. In fact, this work focuses on comparing the proposed sensor configuration and that used in [39]. So, we took care to realize the plasmonic chip in a similar way to that used in [39], as has been described in this section.

\subsection{Synthetic Receptor Film}

\subsubsection{Chemicals}

Reagents: $N, N^{\prime}$-methylene bisacrylamide (BIS) (CAS 110-26-9), Acrylamide (Aam) (CAS 79-06-1), N-tert-butylacrylamide (TBAm) (CAS 107-58-4), 2-hydroxyethyl methacrylate(HEMA) (CAS 868-77-9), ammonium persulfate (APS) (CAS 7727-54-0), $N, N, N^{\prime}, N^{\prime}-$ tetramethylethylenediamine (TEMED) (CAS 110-18-9, sodium dodecyl sulfate (SDS) (CAS 151-21-3), phosphate buffer solution 1.0 M were from Sigma-Aldrich (Darmstadt, Germany) and used without any further purification. All other chemicals were of analytical reagent grade. The solvent was Milli-Q water.

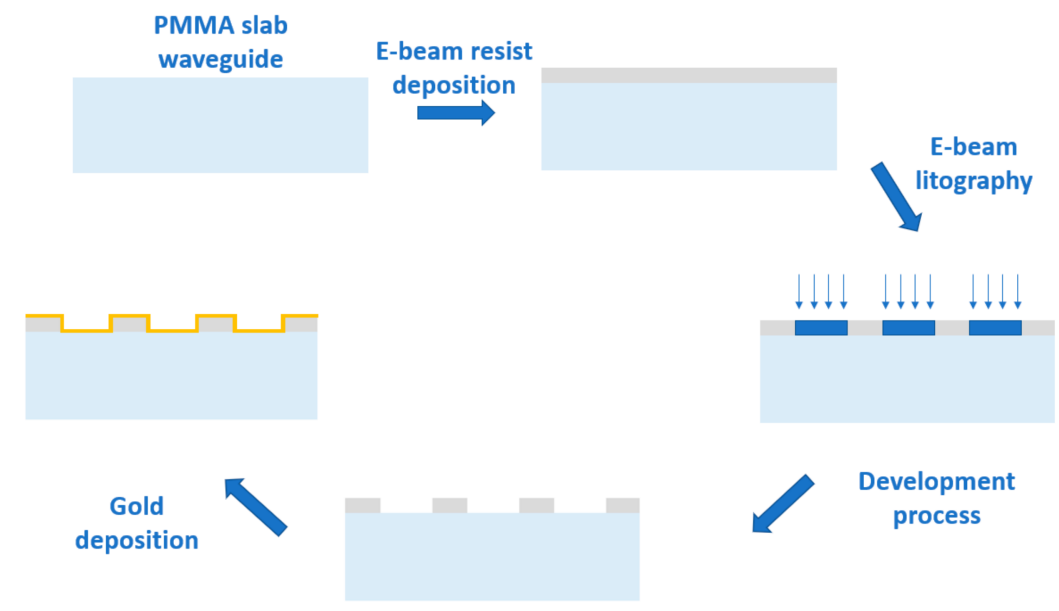

(a)

Figure 1. Cont. 


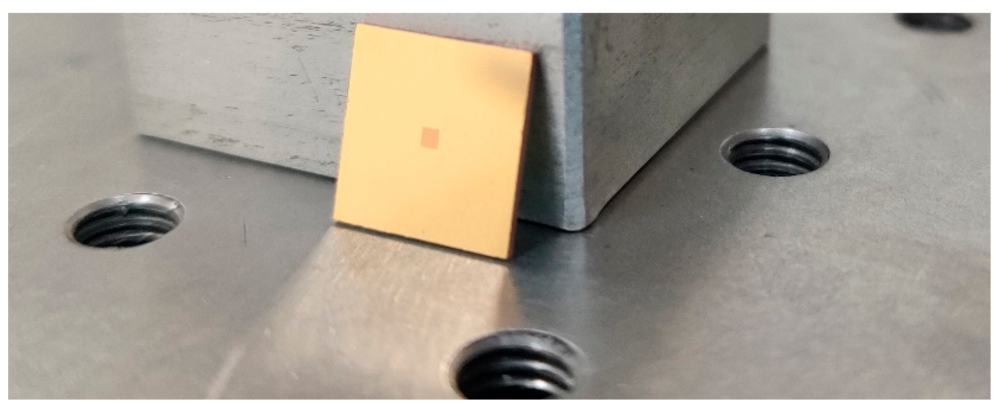

(b)

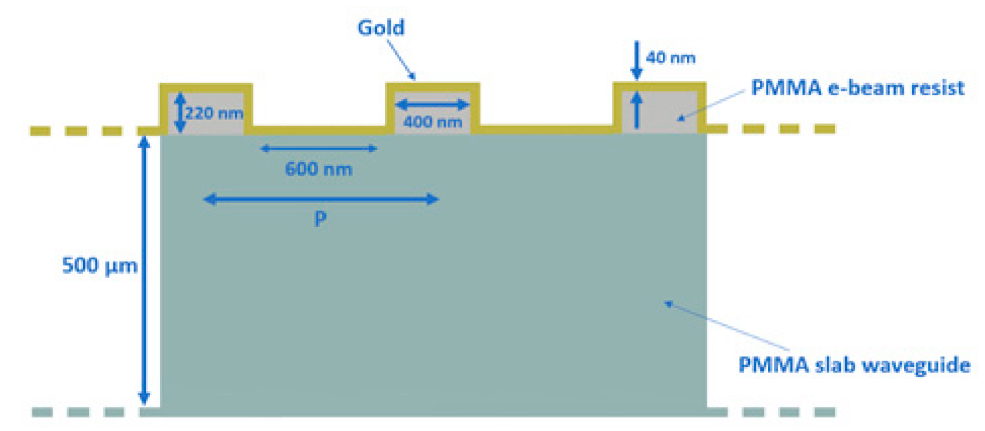

(c)

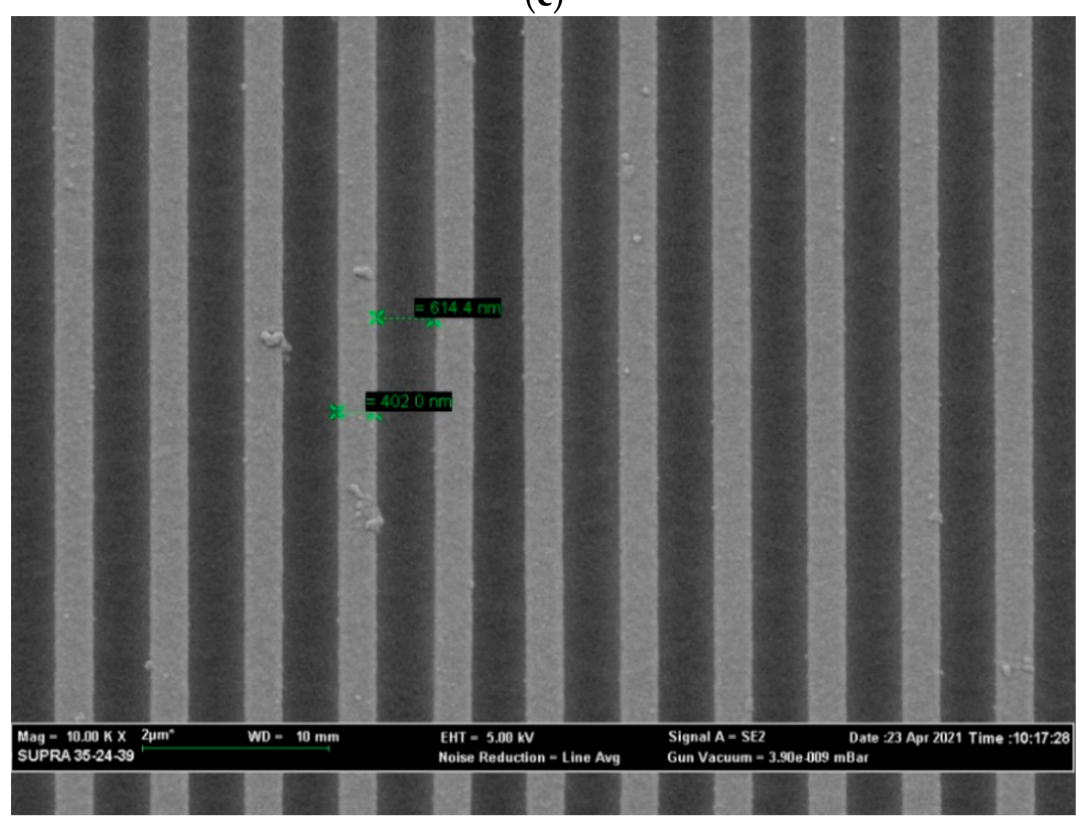

(d)

Figure 1. (a) Outline of the fabrication process of the plasmonic sensor. (b) picture of the PMMA slab waveguide with gold nanograting pattern at the center. (c) Schematic cross section of the examined plasmonic GNG-based chip with the relative dimensions. (d) SEM image of the fabricated nanograting.

The bovine serum albumin (CAS 9048-46-8) and trypsin (CAS 9002-07-7) were from Sigma-Aldrich (Darmstadt, Germany).

\subsubsection{Molecularly Imprinted Polymer for BSA Detection}

Molecularly imprinted polymers (MIPs) are synthetic receptors able to recognize specific molecules or a class of molecules [42,43]. The synthesis is based on using a template molecule and appropriate functional monomers, which coordinate the target molecule by establishing interactions of various kinds (van-der Waals, ionic, dipole-dipole, etc.), forming a complex. 
Subsequently, a cross-linking reagent fixes the complex, forming a polymer around it. At the end of the polymerization process, the template is removed, leaving the interaction sites free and able to reversibly recognize the analyte of interest.

In this study, a synthetic receptor was grown on the Au surface in nanometric film. In particular, a recently developed molecular imprinted polymer synthesis strategy for proteins, under non-denaturing conditions, is used [44]. The preparation is reported below.

With the purpose of covalently binding the polymeric receptor to the gold layer, the optical transducer was first modified with an allyl thiol. In particular, the gold surface of the transducer was immersed in a $10 \% v / v$ solution of allyl thiol in $80 \% v / v$ ethanol solution and $10 \% v / v$ water for $12 \mathrm{~h}$. Subsequently, the platform was washed with Milli-Q water (flushing $3 \mathrm{~mL} 5$ times). This process formed a self-assembled monolayer with a terminal allyl group.

The monomer mixture was prepared by adding Acrylamide (Aam), N-t-butylacrylamide (TBAm), 2-hydroxyethyl methacrylate (HEMA) at 1:0.5:0.6 molar ratio, in a 15-mM phosphate buffer (PB) pH 7.4. Then, $N, N^{\prime}$-methylene bisacrylamide (BIS) was added to the monomeric mix with a final concentration of $0.19 \mathrm{M}$. The pre-polymeric mixture was dispersed by sonication (sonic bath model VWR USC200T) for $10 \mathrm{~min}$ and bubbled with N2 for $30 \mathrm{~min}$ at room temperature. The template (BSA) was added to the pre-polymeric mixture to the final concentration of $1 \mu \mathrm{M}$. Then APS $(0.08 \% w / v)$ and TEMED $(0.06 \% w / v)$ were added. About $2 \mu \mathrm{L}$ of the pre-polymeric mixture was dropped over the sensing region and let polymerize for $15 \mathrm{~min}$ at room temperature, after which the reticulation process was stopped by washing the sensor surface with Milli-Q water. Finally, the template was removed by incubating trypsin $4.2 \times 10^{-8} \mathrm{M}$ for $2 \mathrm{~h}$ at room temperature on the sensor surface and then by washing with an SDS 5\% $(w / v)$ solution. We used an MIP for BSA to compare the results obtained in this work with those carried out in $[42,43]$.

\subsection{Experimental Setup}

In this work, to monitor the GNG-based sensor configuration, we used an experimental setup that exploits the PMMA substrate, acting as a slab waveguide, to excite the nanoplasmonic phenomenon. It consists of a white light source (HL-2000-LL, manufactured by Ocean Optics, Dunedin, FL, USA, with an emission range from $360 \mathrm{~nm}$ to $1700 \mathrm{~nm}$ ), a spectrometer (FLAME-S-VIS-NIR-ES, manufactured by Ocean Optics, Dunedin, FL, USA, with a detection range from $350 \mathrm{~nm}$ to $1023 \mathrm{~nm}$ ), two POF patches, and an aluminum holder. We did not control the light's polarization in this setup because we used a multimode optical waveguide and a trench of air to couple the light in the waveguide.

As shown in Figure 2a, all these components are connected similarly to SPR sensors [14]. Figure $2 \mathrm{~b}$ shows the light propagation path through the setup. In particular, the light is launched from the source to a first POF patch ( $1 \mathrm{~mm}$ total diameter). At the end of the POF, a trench of air is realized in the metallic holder, which can be used to enlarge the number of angles useful to excite plasmons in the nanostructured slab waveguide of PMMA-gold. On the other hand, another POF patch (1 $\mathrm{mm}$ total diameter) kept at the end of the PMMA-gold waveguide, at a $90^{\circ}$ angle with respect to the air trench, collects the transmitted light through nanostructured slab waveguide of PMMA-gold to direct it towards the spectrometer. 


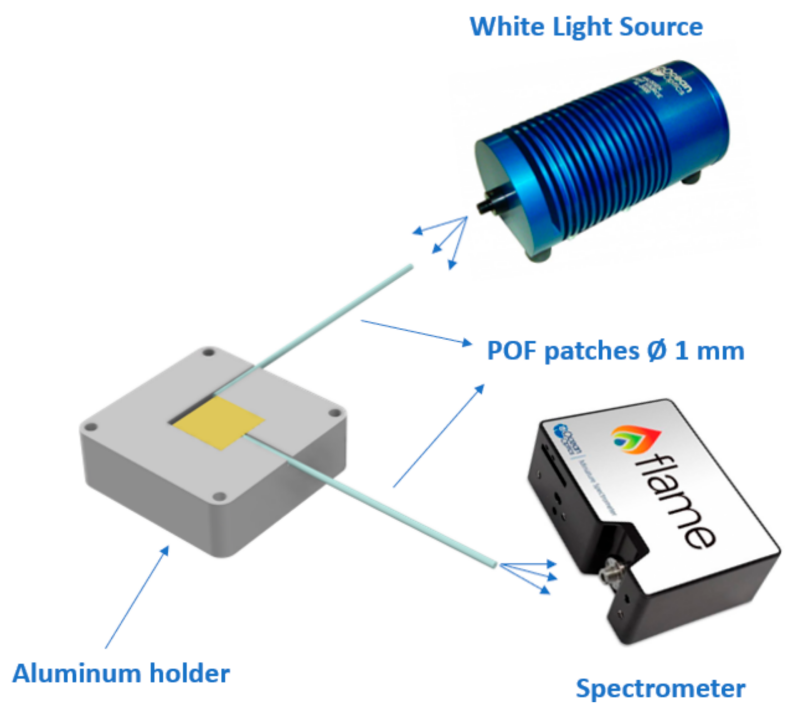

(a)

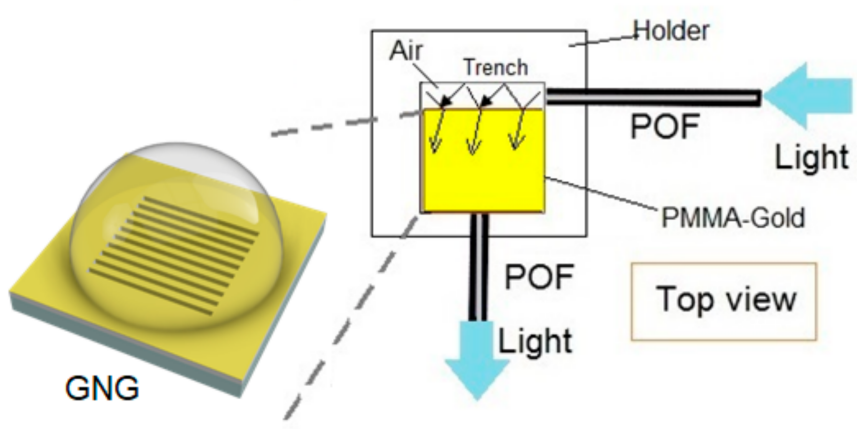

(b)

Figure 2. (a) Outline of the experimental setup. (b) Light propagation path through the setup.

\section{Results}

In the considered setup, in the nano-patterned slab waveguide covered by gold nanofilm and MIP receptor layer, two different plasmonic phenomena, on the sensor surfaces interested in the functionalization process, can be excited.

We deposited the biomimetic receptor on a region larger than the GNG area $\left(1 \mathrm{~mm}^{2}\right)$. So, we had a receptor layer on the GNG surface and the nearby regions, where a continuous gold film was present, as schematically shown in Figure 3. Therefore, when considering the GNG structures, as already underlined in [39], plasmonic hybrid modes are excited due to the mutual interaction between the surface plasmons (SPs) and the localized surface plasmons (LSPs). In contrast, the classic SPR phenomenon occurs where the continuous gold film is present.

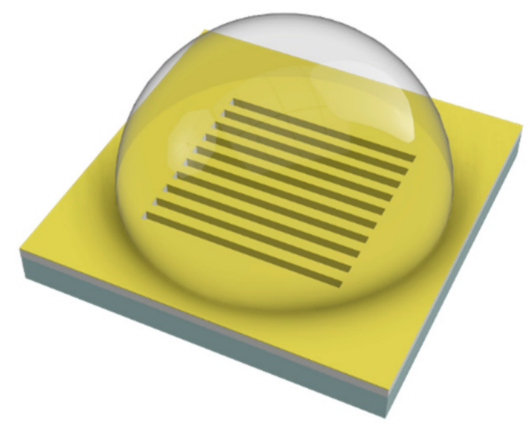

Figure 3. Outline of the functionalization process: the pre-polymeric mixture is dropped on the sensor surface and covers both the nanograting and the nearby regions. 
In Ref. [39], there was evidence only of the first kind of plasmonic phenomenon (hybrid modes) since the direction of the input light was orthogonal with respect to the sensor surface, and this working condition does not allow the excitation of SPs on the continuous gold film nearby the nanograting.

Moreover, when interrogating the same plasmonic GNG-based sensor using the experimental setup reported in [39], neither the resonance wavelength areas nor the optical performances were influenced by the nanostripes' orientation with respect to the direction of the input light.

In contrast to the above, we determined that these aspects have particular relevance in the sensor configurations proposed here. For this reason, as schematically shown in Figure 4, we identified two possible sensor configurations, that is, "GNG-based longitudinal", where the nanostripes are located along the same direction of the input light (see Figure 4a), and "GNG-based orthogonal", when the two mentioned directions are mutually orthogonal (see Figure $4 b$ ).

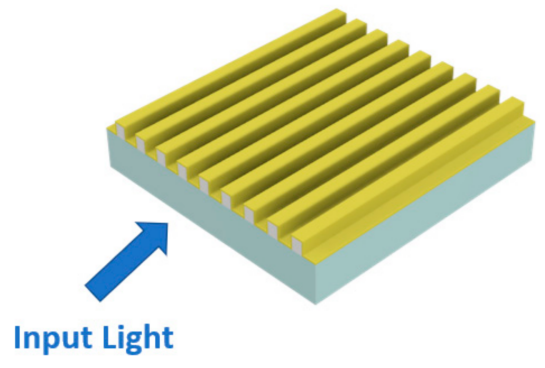

(a)

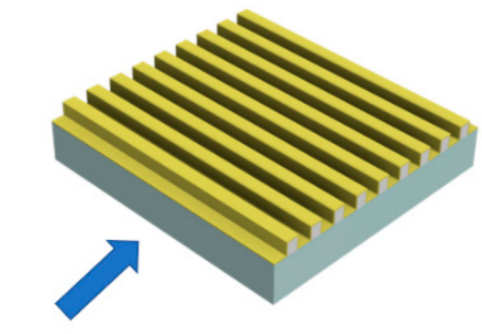

Input Light

(b)

Figure 4. (a) "GNG-based longitudinal" configuration. (b) "GNG-based orthogonal" configuration.

Figure 5a reports the plasmonic spectra relative to the "GNG-based longitudinal" configuration at different BSA concentrations. These spectra were obtained by normalizing the transmitted spectra to the one obtained with air as the surrounding medium. This kind of normalization was possible in this setup because we had no evidence of resonance in air, in contrast to what is shown in [39]. As shown in the zooms of Figure 5, reported in Figure $5 b, c$, two distinct resonance phenomena can be observed at around $550 \mathrm{~nm}$ (plasmonic hybrid modes) and $630 \mathrm{~nm}$ (SPR).

In the considered case, by analyzing more in detail these two plasmonic resonances, it is clear that they are sensitive to a different range of BSA concentrations.

In particular, at low concentrations $\left(10^{-10} \mathrm{M}-10^{-7} \mathrm{M}\right)$, the resonance wavelength peak at $550 \mathrm{~nm}$ decreases (blue shift) when the BSA concentration increases, as shown in Figure $5 \mathrm{~b}$. On the opposite, in a different BSA range $\left(10^{-6} \mathrm{M}-10^{-5} \mathrm{M}\right)$ the resonance wavelength peak at $630 \mathrm{~nm}$ increases (red shift) when the analyte concentration increases too (see Figure 5c). The first behavior (blue shift at around $500 \mathrm{~nm}$ ) is similar to the one observed in [39]. It can be ascribed to the hybrid modes excited by the nanograting, even if at different resonance conditions. The second one (red shift at around $650 \mathrm{~nm}$ ) is typical when considering SPR phenomenon.

Conversely, when considering the "GNG-based orthogonal" configuration, only a resonance peak at $630 \mathrm{~nm}$ is visible, as shown in Figure 6a. In particular, at increasing BSA concentration, the resonance wavelength decreases similarly to what we observed with the resonance peak at $550 \mathrm{~nm}$ in the "GNG-based longitudinal" configuration. Figure 6b shows a zoom of the resonance wavelength region.

This aspect could be explained by the fact that a 90-degree-rotation of the pattern causes a wavelength overlap of the two previously mentioned plasmonic phenomena. In fact, in our previous work [45], we demonstrated, by performing numerical simulations in a 
similar structure, that when considering an orthogonal configuration, the GNG's resonance wavelength area shifts towards higher values with respect to the longitudinal one [45].

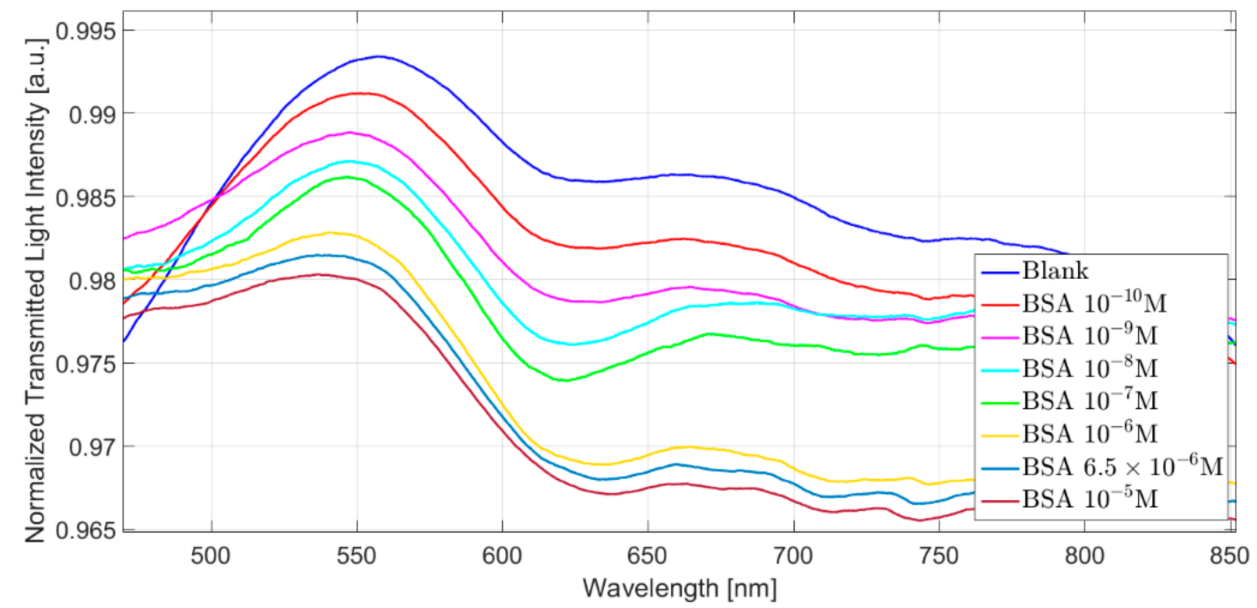

(a)

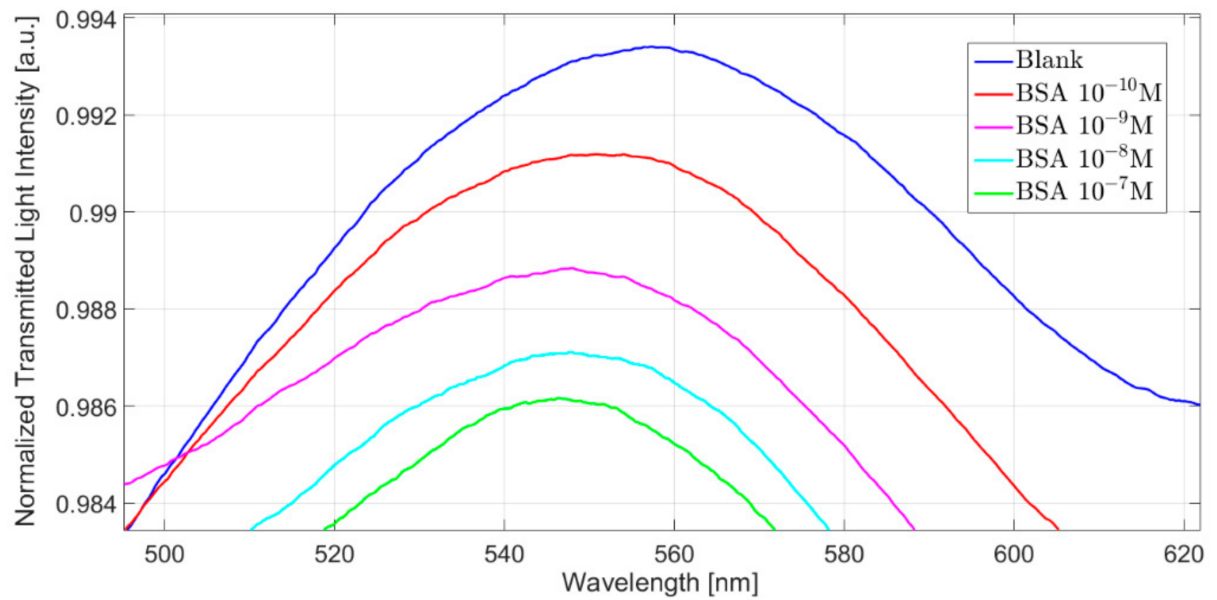

(b)

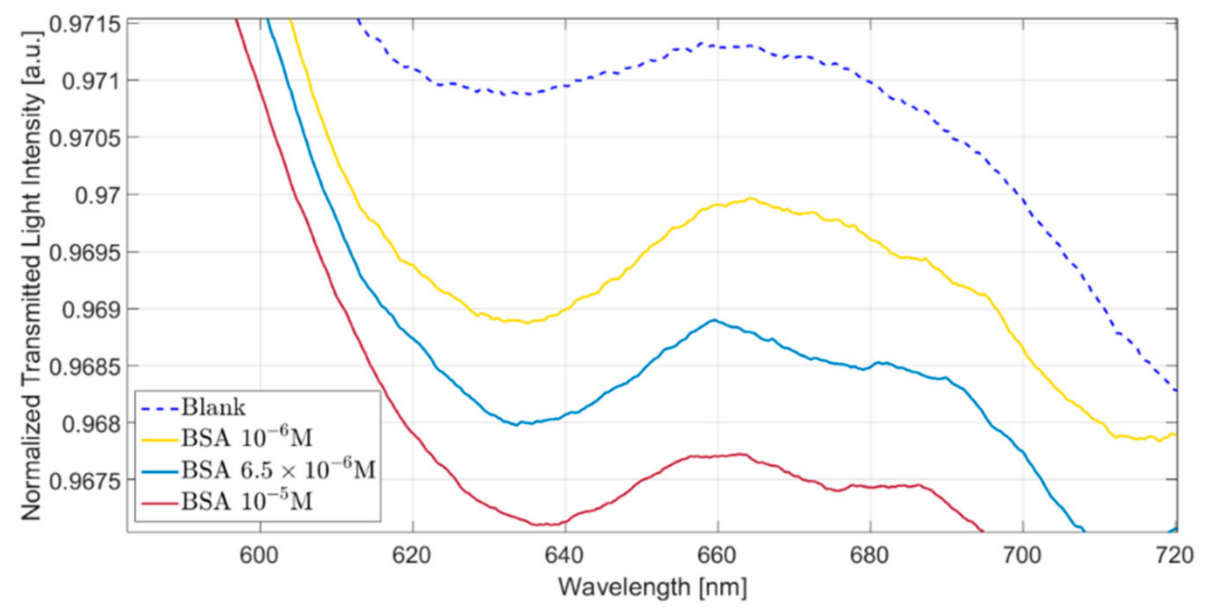

(c)

Figure 5. (a) Plasmonic spectra at varying of the BSA concentration $\left(0-10^{-5} \mathrm{M}\right)$ relative to "GNGbased longitudinal" configuration. Zoom of the resonance wavelength area relative to (b) peak at $550 \mathrm{~nm}$ and (c) peak at $630 \mathrm{~nm}$. 


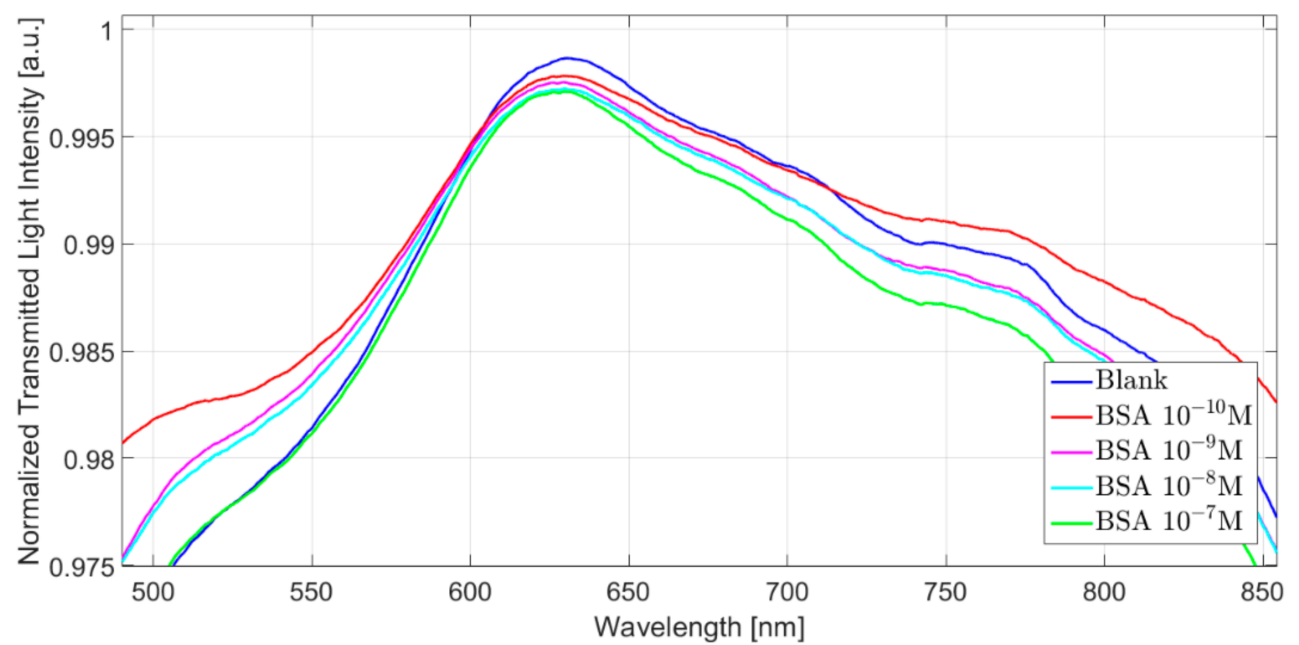

(a)

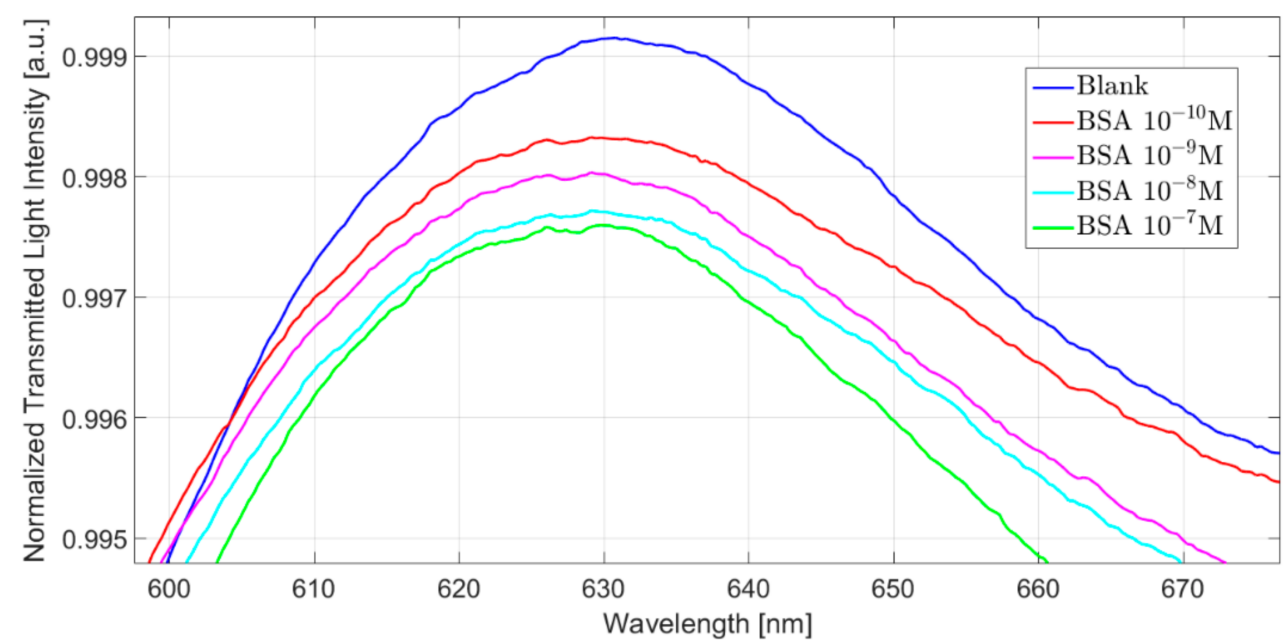

(b)

Figure 6. (a) Plasmonic spectra at varying of the BSA concentration $\left(0-10^{-7} \mathrm{M}\right)$ relative to "GNGbased orthogonal" configuration. (b) Zoom of the resonance wavelength area.

The observed resonance could be ascribable to the GNG (i.e., the blue-shift one) because it is predominant over the other one, even if it is negatively influenced by the SPR phenomenon, which goes in the opposite direction. This mutual destructive interference between these different plasmonic phenomena (blue shift and red shift) is confirmed by a worse optical sensitivity. The orthogonal configuration denotes a minor total shift in resonance wavelength between the blank and higher BSA concentrations.

\section{Discussion}

Figure 7 reports the dose-response curves carried out for the BSA binding test measurements, along with the Hill fitting of the experimental values and the error bars, for both the analyzed configurations (longitudinal and orthogonal). In particular, Figure 7a shows the BSA detection relative to the blue shift resonances, whereas the detection carried out by the red shift resonance is reported in Figure 7b. In Figure 7, each experimental value was calculated as the absolute value variation with respect to the blank solution (i.e., a solution without the analyte). The Hill equation used to fit the experimental data is reported as follows:

$$
\left|\Delta \lambda_{c}\right|=\left|\lambda_{\mathrm{c}}-\lambda_{0}\right|=\Delta \lambda_{\max } \mid \cdot\left(\mathrm{c}^{\mathrm{n}} /\left(\mathrm{K}^{\mathrm{n}}+\mathrm{c}^{\mathrm{n}}\right)\right)
$$


where $\mathrm{c}$ is the analyte concentration, $\lambda_{\mathrm{c}}$ is the resonance wavelength at the concentration $c, \lambda_{0}$ is the resonance wavelength at zero concentration (blank), $\Delta \lambda_{\max }$ is the maximum value of $\Delta \lambda_{c}$ (calculated by the saturation value minus the blank value, i.e., $\lambda_{\max }-\lambda_{0}$ ). The parameters $n$ and $K$ are the Hill constants.

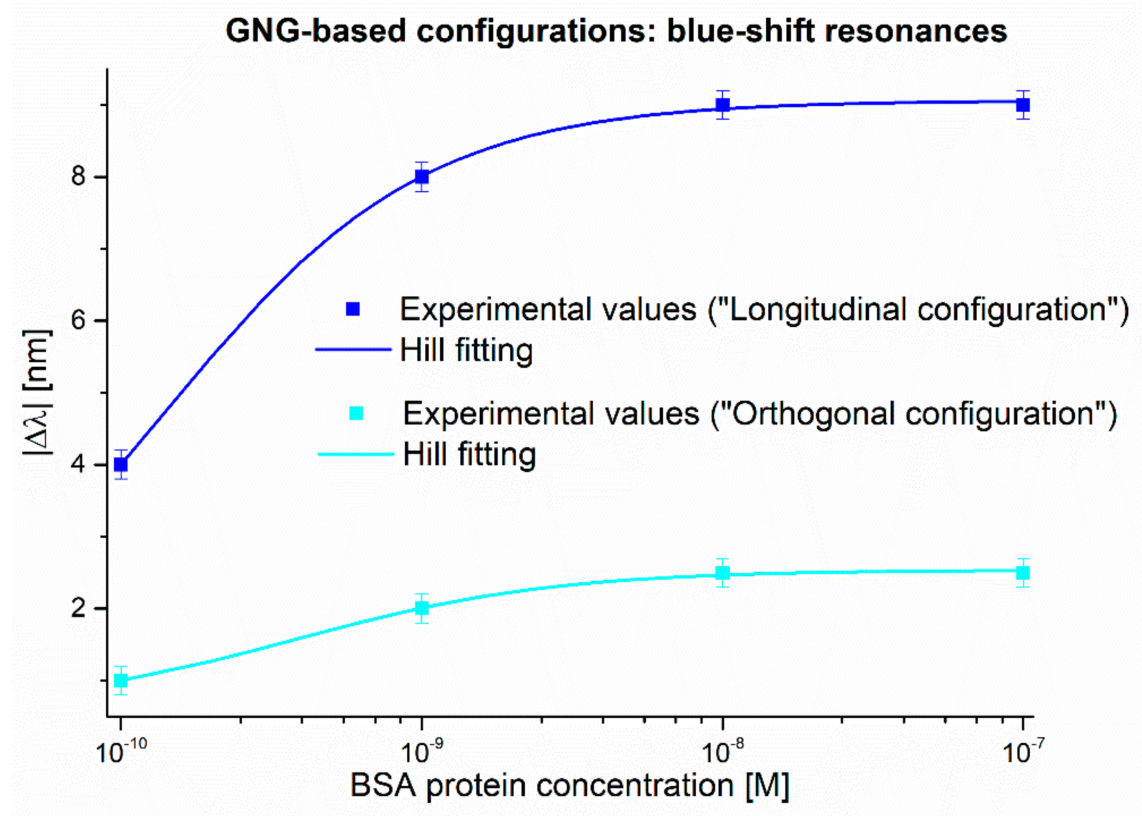

(a)

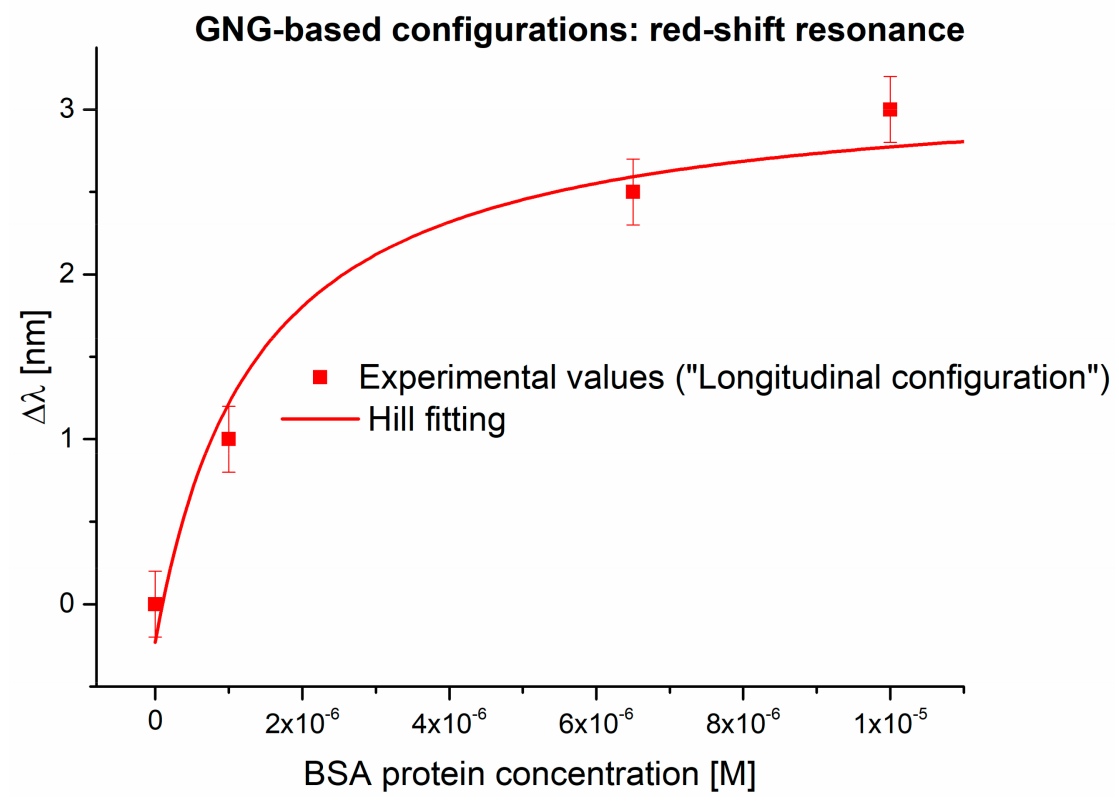

(b)

Figure 7. BSA dose-response curves with Hill fitting of the data and error bars relative to (a) GNGbased longitudinal and GNG-based orthogonal blue-shift resonances and (b) GNG-based longitudinal red-shift resonance.

Table 1 reports the parameters relative to Equation (1) for each analyzed sensor configuration and each plasmonic phenomenon. Table 2 reports the chemical parameters calculated according to Equation (1) and considering $n=1$ (in this case, the Hill equation is the same as the Langmuir equation). At low concentration, which means $c$ much lower than $K$, Equation (1) can be approximated to a linear function, where the slope $\left(\Delta \lambda_{\max } / K\right)$ 
is called "sensitivity at low concentration". It is essential to underline that, to compare the limit of detection (LOD) with other SPR POF-based sensor configurations, we used the same LOD definition adopted in $[39,44]$. So, by considering the linear behavior at low BSA concentration, the latter is used to calculate the LOD defined as:

$$
\operatorname{LOD}=\left(2 \cdot \sigma_{\lambda_{0}}\right) / \frac{\Delta \lambda_{\max }}{\mathrm{K}}
$$

where $\sigma_{\lambda_{0}}$ represents the standard error relative to the blank solution, that is, the standard error relative to $\lambda_{0}$ reported in Table 1 . In Table 2, the sensitivity at low concentration, the affinity constant (Kaff), and the LOD are reported for the tested sensor configurations and the different plasmonic phenomena.

Table 1. Hill fitting parameters relative to Equation (1) for different sensor configurations.

\begin{tabular}{|c|c|c|c|c|c|c|c|c|c|}
\hline \multirow[t]{2}{*}{ Configuration } & \multicolumn{2}{|c|}{$\lambda_{0}(\mathrm{~nm})$} & \multicolumn{2}{|c|}{$\lambda_{\max }(\mathrm{nm})$} & \multicolumn{2}{|c|}{ K (M) } & \multirow{2}{*}{$\begin{array}{c}n \\
\text { Value }\end{array}$} & \multicolumn{2}{|c|}{ Statistics } \\
\hline & Value & Stand. Err. & Value & Stand. Err. & Value & Stand. Err. & & Reduced Chi-Sqr & Adj. R-Square \\
\hline $\begin{array}{l}\text { GNG-based longitudinal } \\
\text { (blue shift) }\end{array}$ & 0.30 & 0.73 & 9.06 & 0.06 & $1.37 \times 10^{-10}$ & $2.65 \times 10^{-11}$ & 1 & 0.14 & 0.99 \\
\hline $\begin{array}{l}\text { GNG-based orthogonal } \\
\text { (blue shift) }\end{array}$ & 0.59 & 0.11 & 2.54 & 0.03 & $3.74 \times 10^{-10}$ & $7.56 \times 10^{-11}$ & 1 & 0.05 & 0.99 \\
\hline $\begin{array}{l}\text { GNG-based longitudinal } \\
\text { (red shift) }\end{array}$ & -0.23 & 0.69 & 3.18 & 0.35 & $1.35 \times 10^{-6}$ & $6.57 \times 10^{-7}$ & 1 & 1.17 & 0.98 \\
\hline
\end{tabular}

Table 2. Chemical parameters relative to the BSA detection for the tested sensor configurations.

\begin{tabular}{|c|c|c|c|c|}
\hline \multirow[t]{2}{*}{ Configuration } & \multirow[b]{2}{*}{$\begin{array}{c}\text { Plasmonic } \\
\text { Phenomenon }\end{array}$} & \multicolumn{3}{|c|}{ Parameter } \\
\hline & & $\begin{array}{c}\text { Sensitivity at Low } \\
\text { Concentration }(\mathrm{nm} / \mathrm{M})\end{array}$ & LOD [M] & $K_{\mathrm{aff}}=1 / K\left(M^{-1}\right)$ \\
\hline GNG-based longitudinal (blue shift) & Hybrid & $6.39 \times 10^{10}$ & $2.3 \times 10^{-11}$ & $7.3 \times 10^{9}$ \\
\hline GNG-based orthogonal (blue shift) & Hybrid & $5.2 \times 10^{9}$ & $4.2 \times 10^{-11}$ & $2.7 \times 10^{9}$ \\
\hline GNG-based longitudinal (red shift) & SPR & $2.53 \times 10^{6}$ & $5.4 \times 10^{-7}$ & $7.4 \times 10^{5}$ \\
\hline
\end{tabular}

Finally, a comparative analysis relative to the BSA detection was reported in Table 3 regarding the LOD and the detection range. We compared these results with the ones reported in $[39,44]$, where the same MIP receptor was used.

Table 3. Comparative analysis in terms of limit of detection and BSA detection range for several plasmonic configurations combined with the same MIP receptor.

\begin{tabular}{cccc}
\hline Configuration & LOD & BSA Detection Range & Reference \\
\hline Gold nanograting on a PMMA substrate & $37 \mathrm{pM}$ & $37 \mathrm{pM}-100 \mathrm{nM}$ & {$[39]$} \\
(same chip of this work in a different setup) & $0.37 \mu \mathrm{M}$ & $0.37 \mu \mathrm{M}-6.5 \mu \mathrm{M}$ & [44] \\
SPR D-shaped POFs & $23 \mathrm{pM}$ & $23 \mathrm{pM}-10 \mathrm{nM}$ & This work \\
GNG-based longitudinal (blue-shift resonance) & $0.54 \mu \mathrm{M}$ & $0.54 \mu \mathrm{M}-10 \mu \mathrm{M}$ & This work \\
GNG-based longitudinal (red-shift resonance) & $42 \mathrm{pM}$ & $42 \mathrm{pM}-10 \mathrm{nM}$ & This work \\
GNG-based orthogonal (blue-shift resonance) & & \\
\hline
\end{tabular}

As it can be seen, we first confirmed that nanostructured sensors' use leads to a significant improvement when considering biosensing applications, as already underlined in [39]. Moreover, we demonstrated that when we use the PMMA as a slab waveguide or as a transparent substrate, the biosensing performances at very low analyte concentrations $(\mathrm{pM}-\mathrm{nM})$ are similar. We obtained a similar LOD in both the analyzed configurations (longitudinal and orthogonal) with respect to the one obtained in [39]. The advantage of using the sensing approach adopted in this work is the possibility to monitor a wide range of analyte concentrations using a single plasmonic sensor. In fact, by exploiting the "GNG-based longitudinal" configuration, we widened the detection range by taking advantage of two distinct plasmonic phenomena. In particular, the blue shift resonance is sensitive in the BSA range between $23 \mathrm{pM}$ (LOD) and $10 \mathrm{nM}$ (saturation value). In contrast, 
the red-shift resonance is sensitive in the BSA range between $0.54 \mu \mathrm{M}$ (LOD) and $10 \mu \mathrm{M}$ (saturation value). These values, relative to the red-shift resonance, are similar to those obtained with the SPR-POF platform [44], where an LOD equal to $0.37 \mu \mathrm{M}$ was obtained.

\section{Conclusions}

A specific experimental setup has been used to monitor a nanoplasmonic slab waveguide functionalized with a synthetic receptor for BSA detection. In particular, we have determined the influence of the nanostripes' orientation with respect to the input light direction by identifying two possible sensor configurations, orthogonal and longitudinal. Both configurations have shown blue-shift resonances at increasing the target analyte concentrations, obtaining a similar LOD $(\approx \mathrm{pM})$ if compared to the one obtained with the same chip interrogated with a different experimental setup [39]. In addition, the longitudinal configuration has also denoted a red-shift resonance, ascribable to an SPR phenomenon, sensitive to higher BSA concentrations. In the latter case, exploiting the SPR phenomenon, we obtained an LOD $(\cong 0.57 \mu \mathrm{M})$ similar to the one obtained with an SPR-POF reference sensor functionalized with the same receptor [44]. Standing this, the more evident advantage of the proposed sensing approach is the feasibility of monitoring a wide range of analyte concentrations (from $\mathrm{pM}$ to $\mu \mathrm{M}$ ) by a single plasmonic platform (longitudinal configuration).

As a final remark, it is essential to underline that in this kind of experimental setup, a greatly reduced volume of solution (about $10 \mu \mathrm{L}$ ) is required, compared to the $1 \mathrm{~mL}$ used in the setup described in [39].

Author Contributions: Conceptualization, F.A., L.Z., and N.C.; methodology, F.A., A.M., G.C., C.P., L.Z., G.D., and N.C.; validation, F.A., A.M., G.C., C.P., L.Z., G.D., G.P., and N.C.; formal analysis, F.A., A.M., G.C., C.P., L.Z., G.D., G.P., C.E., S.D.R., and N.C.; investigation, F.A., A.M., G.C., C.P., L.Z., G.D., G.P., C.E., S.D.R., and N.C.; resources, N.C., G.P., and L.Z.; data curation, F.A., A.M., G.C., C.P., L.Z., G.D., G.P., and N.C.; writing—original draft preparation, F.A., A.M., G.C., C.P., L.Z., G.D., G.P., C.E., S.D.R., and N.C.; writing-review and editing, F.A., A.M., G.C., C.P., L.Z., G.D., G.P., C.E., S.D.R., and N.C.; supervision, N.C. and L.Z. All authors have read and agreed to the published version of the manuscript.

Funding: This research received no external funding.

Data Availability Statement: The data is available on reasonable request from the corresponding author.

Acknowledgments: This work was supported by the VALERE program of the University of Campania "Luigi Vanvitelli" (Italy), Campania project.

Conflicts of Interest: The authors declare no conflict of interest.

\section{References}

1. Barshilia, D.; Chau, L.-K.; Chang, G.-E. Low-cost planar waveguide-based optofluidic sensor for real-time refractive index sensing. Opt. Express 2020, 28, 27337-27345. [CrossRef]

2. Mukundan, H.; Anderson, A.S.; Grace, W.K.; Grace, K.M.; Hartman, N.; Martinez, J.S.; Swanson, B.I. Waveguide-Based Biosensors for Pathogen Detection. Sensors 2009, 9, 5783. [CrossRef]

3. Fuentes, O.; Corres, J.M.; Domínguez, I.; del Villar, I.; Matias, I.R. Simultaneous Measurement of Refractive Index and Temperature using LMR on planar waveguide. In Proceedings of the 2020 IEEE SENSORS, Rotterdam, The Netherlands, 25-28 October 2020; pp. 1-4.

4. Horváth, R.; Pedersen, H.C.; Skivesen, N.; Selmeczi, D.; Larsen, N.B. Optical waveguide sensor for on-line monitoring of bacteria. Opt. Lett. 2003, 28, 1233-1235. [CrossRef]

5. Hulme, J.P.; Goddard, N.J.; Lu, C. Simple leaky-waveguide devices for the detection of bacterial spores. Sens. Actuators B Chem. 2011, 160, 1508-1513. [CrossRef]

6. Schmitt, K.; Schirmer, B.; Hoffmann, C.; Brandenburg, A.; Meyrueis, P. Interferometric biosensor based on planar optical waveguide sensor chips for label-free detection of surface bound bioreactions. Biosens. Bioelectron. 2011, 22, 2591-2597. [CrossRef]

7. Wang, M.; Uusitalo, S.; Liedert, C.; Hiltunen, J.; Hakalahti, L.; Myllylä, R. Polymeric dual-slab waveguide interferometer for biochemical sensing applications. Appl. Opt. 2012, 51, 1886-1893. [CrossRef]

8. Lončar, M.; Doll, T.; Vučković, J.; Scherer, A. Design and Fabrication of Silicon Photonic Crystal Optical Waveguides. J. Light. Technol. 2000, 18, 1402. [CrossRef] 
9. Gao, L.; Yang, X.; Shu, Y.; Chen, X.; Wang, J. Ionic liquid-based slab optical waveguide sensor for the detection of ammonia in human breath. J. Colloid Interface Sci. 2018, 512, 819-825. [CrossRef]

10. Shinbo, K.; Mizusawa, K.; Takahashi, H.; Ohdaira, Y.; Baba, A.; Kato, K.; Kaneko, F.; Miyadera, N. Vapor Sensing Using Waveguide-Based Multiple Surface Plasmon Resonance Sensors. Jpn. J. Appl. Phys. 2011, 50, 01BC15. [CrossRef]

11. Harris, R.D.; Wilkinson, J.S. Waveguide surface plasmon resonance sensors. Sens. Actuators B Chem. 1995, 29, 261-267. [CrossRef]

12. Huang, J.-G.; Lee, C.-L.; Lin, H.-M.; Chuang, T.-L.; Wang, W.-S.; Juang, R.-H.; Wang, C.-H.; Lee, C.K.; Lin, S.-M.; Lin, C.-W. A miniaturized germanium-doped silicon dioxide-based surface plasmon resonance waveguide sensor for immunoassay detection. Biosens. Biolectron. 2006, 22, 519-525. [CrossRef] [PubMed]

13. Cennamo, N.; Trigona, C.; Graziani, S.; Zeni, L.; Arcadio, F.; Di Pasquale, G.; Pollicino, A. An Eco-Friendly Disposable Plasmonic Sensor Based on Bacterial Cellulose and Gold. Sensors 2019, 19, 4894. [CrossRef] [PubMed]

14. Cennamo, N.; Mattiello, F.; Zeni, L. Slab Waveguide and Optical Fibers for Novel Plasmonic Sensor Configurations. Sensors 2017, 17, 1488. [CrossRef]

15. Baryshev, A.V.; Merzlikin, A.M.; Inoue, M. Efficiency of optical sensing by a plasmonic photonic-crystal slab. J. Phys. D Appl. Phys. 2013, 46, 125107. [CrossRef]

16. Chen, J.; Yuan, J.; Zhang, Q.; Ge, H.; Tang, C.; Liu, Y.; Guo, B. Dielectric waveguide-enhanced localized surface plasmon resonance refractive index sensing. Opt. Mater. Express 2018, 8, 342-347. [CrossRef]

17. Zheng, G.; Zou, X.; Chen, Y.; Xu, L.; Rao, W. Fano resonance in graphene-MoS2 heterostructure-based surface plasmon resonance biosensor and its potential applications. Opt. Mater. 2017, 66, 171-178. [CrossRef]

18. Consani, C.; Ranacher, C.; Tortschanoff, A.; Grille, T.; Irsigler, P.; Jakoby, B. Mid-infrared photonic gas sensing using a silicon waveguide and an integrated emitter. Sens. Actuators B Chem. 2018, 274, 60-65. [CrossRef]

19. Huang, Y.; Kalyoncu, S.K.; Zhao, Q.; Torun, R.; Boyraz, O. Silicon-on sapphire waveguides design for mid-IR evanescent field absorption gas sensors. Opt. Commun. 2014, 313, 186-194. [CrossRef]

20. Siebert, R.; Müller, J. Infrared integrated optical evanescent field sensor for gas analysis: Part I: System design. Sens. Actuators A Phys. 2005, 119, 138-149. [CrossRef]

21. Airoudj, A.; Debarnot, D.; Bêche, B.; Poncin-Epaillard, F. Design and sensing properties of an integrated optical gas sensor based on a multilayer structure. Anal. Chem. 2008, 80, 9188-9194. [CrossRef]

22. Maurya, J.B.; Prajapati, Y.K.; Raikwar, S.; Saini, J.P. A silicon-black phosphorous based surface plasmon resonance sensor for the detection of $\mathrm{NO}_{2}$ gas. Optik 2018, 160, 428-433. [CrossRef]

23. Ge, D.; Chen, H.; Jin, P.; Zhang, L.; Li, W.; Jiao, J. Magnetic field sensor based on evanescent wave coupling effect of photonic crystal slab microcavity. J. Magn. Magn. Mater. 2021, 527, 167696. [CrossRef]

24. Deghdak, R.; Bouchemat, M.; Lahoubi, M.; Pu, S.; Bouchemat, T.; Otmani, H. Sensitive magnetic field sensor using 2D magnetic photonic crystal slab waveguide based on BIG/GGG structure. J. Comput. Electron. 2017, 16, 392-400. [CrossRef]

25. Kozma, P.; Kehl, F.; Ehrentreich-Förster, E.; Stamm, C.; Bier, F.F. Integrated planar optical waveguide interferometer biosensors: A comparative review. Biosens. Bioelectron. 2014, 58, 287-307. [CrossRef]

26. Densmore, A.; Xu, D.-X.; Waldron, P.; Janz, S.; Delâge, A.; Lopinski, G.; Mischki, T.; Cheben, P.; Post, E.; Lapointe, J.; et al. Label-free biosensing using silicon planar waveguide technology. In Photonics North 2007; SPIE: Bellingham, WA, USA, 2007; Volume 6796, p. 67962.

27. Kumar, M.; Muniswamy, V.; Guha, K.; Iannacci, J.; Krishnaswamy, N. Analysis of integrated silicon nitride lab-on-a-chip optofluidic sensor at visible wavelength for absorbance based biosensing applications. Microsyst. Technol. 2021, 27, 3499-3506. [CrossRef]

28. White, N. Thick Films. In Springer Handbook of Electronic and Photonic Materials; Kasap, S., Capper, P., Eds.; Springer: Cham, Switzerland, 2017.

29. Gasior, K.; Martynkien, T.; Urbanczyk, W. Effect of constructional parameters on the performance of a surface plasmon resonance sensor based on a multimode polymer optical fiber. Appl. Opt. 2014, 53, 8167-8174. [CrossRef]

30. Kanso, M.; Cuenot, S.; Louarn, G. Sensitivity of Optical Fiber Sensor Based on Surface Plasmon Resonance: Modeling and Experiments. Plasmonics 2008, 3, 49-57. [CrossRef]

31. Gupta, B.D.; Verma, R.K. Surface Plasmon Resonance-Based Fiber Optic Sensors: Principle, Probe Designs, and Some Applications. J. Sens. 2009, 2009, 979761. [CrossRef]

32. Dwivedi, Y.S.; Sharma, A.K.; Gupta, B.D. Influence of Design Parameters on the Performance of a Surface Plasmon Sensor Based Fiber Optic Sensor. Plasmonics 2008, 3, 79-86. [CrossRef]

33. Suzuki, H.; Sugimoto, M.; Matsui, Y.; Kondoh, J. Effects of gold film thickness on spectrum profile and sensitivity of a multimodeoptical-fiber SPR sensor. Sens. Actuators B Chem. 2008, 132, 26-33. [CrossRef]

34. Slavik, R.; Homola, J.; Ctyroky, J.; Brynda, E. Novel spectral fiber optic sensor based on surface plasmon resonance. Sens. Actuators B Chem. 2001, 74, 106-111. [CrossRef]

35. Mishra, K.S.; Zou, B.; Chiang, K.S. Surface-Plasmon-Resonance Refractive-Index Sensor with Cu-Coated Polymer Waveguide. IEEE Photonics. Technol. Lett. 2016, 28, 1835-1838. [CrossRef]

36. Walter, J.-G.; Eilers, A.; Alwis, L.S.M.; Roth, B.W.; Bremer, K. SPR Biosensor Based on Polymer Multi-Mode Optical Waveguide and Nanoparticle Signal Enhancement. Sensors 2020, 20, 2889. [CrossRef] [PubMed] 
37. Zeni, L.; Pesavento, M.; Marchetti, S.; Cennamo, N. Slab plasmonic platforms combined with Plastic Optical Fibers and Molecularly Imprinted Polymers for chemical sensing. Opt. Laser Technol. 2018, 107, 484-490. [CrossRef]

38. Cennamo, N.; Massarotti, D.; Conte, L.; Zeni, L. Low Cost Sensors Based on SPR in a Plastic Optical Fiber for Biosensor Implementation. Sensors 2011, 11, 1752. [CrossRef]

39. Arcadio, F.; Zeni, L.; Montemurro, D.; Eramo, C.; Di Ronza, S.; Perri, C.; D’Agostino, G.; Chiaretti, G.; Porto, G.; Cennamo, N. Biochemical sensing exploiting plasmonic sensors based on gold nanogratings and polymer optical fibers. Photonics Res. 2021, 9, 1397-1408. [CrossRef]

40. Chang, Y.-C.; Huang, B.-H.; Lin, T.-H. Surface-Enhanced Raman Scattering and Fluorescence on Gold Nanogratings. Nanomaterials 2020, 10, 776. [CrossRef]

41. Szunerits, S.; Boukherroub, R. Sensing using localised surface plasmon resonance sensors. Chem. Commun. 2012, 48, 8999-9010. [CrossRef]

42. BelBruno, J.J. Molecularly Imprinted Polymers. Chem. Rev. 2019, 119, 94-119. [CrossRef]

43. Haupt, K.; Mosbach, K. Molecularly Imprinted Polymers and Their Use in Biomimetic Sensors. Chem. Rev. 2000, 100, 2495-2504. [CrossRef]

44. Cennamo, N.; D’Agostino, G.; Perri, C.; Arcadio, F.; Chiaretti, G.; Parisio, E.M.; Camarlinghi, G.; Vettori, C.; Di Marzo, F.; Cennamo, R.; et al. Proof of Concept for a Quick and Highly Sensitive On-Site Detection of SARS-CoV-2 by Plasmonic Optical Fibers and Molecularly Imprinted Polymers. Sensors 2021, 21, 1681. [CrossRef] [PubMed]

45. Cennamo, N.; Mattiello, F.; Jorge, P.A.S.; Sweid, R.; De Maria, L.; Pesavento, M.; Zeni, L. Numerical Results on the Exploitation of Gold Nanostructures in Plastic Optical Fibers Based Plasmonic Sensors. In Sensors and Microsystems; AISEM, 2017; Lecture Notes in Electrical, Engineering; Leone, A., Forleo, A., Francioso, L., Capone, S., Siciliano, P., Di Natale, C., Eds.; Springer: Cham, Switzerland, 2017; Volume 457. 